\title{
LINK TO AN INERTIAL SYSTEM
}

\author{
A.N. ARGUE \\ Institute of Astronomy \\ Cambridge $C B 3$ OHA \\ $U K$ \\ and \\ GRAEME L. WHITE \\ Faculty of Science and Technology \\ University of Western Sydney, Nepean \\ P.O. Box 10 N.S.W. 2750 \\ Australia
}

\begin{abstract}
The homogeneous positional reference frame to be established by Hipparcos must be brought into coincidence with the new IAU frame based on extragalactic radio sources. A review is given of the methods by which this is to be achieved.
\end{abstract}

\section{Introduction}

It is planned that Hipparcos will stop making measurements early in 1993, and for the data analysis consortia NDAC and FAST to have completed their tasks during 1995. The final catalogues derived by the two consortia are not expected to differ significantly, so that they can therefore be combined into one catalogue. This will be a highly homogeneous and rigid global frame with six degrees of freedom corresponding to orientation and inertial rotation which will be fixed provisionally by reference to the FK5.

If by this stage the radio interferometric observations to be described below are in a satisfactory state, the catalogue will be further refined; if not, it will be issued as it stands, as 'the Hipparcos Catalogue'.

The FK5 frame contains global irregularities running up to $0^{\prime \prime} .01$ (Morrison et al. (1991)), and while these zonal errors will not reappear in the Hipparcos Catalogue which will remain highly homogeneous and rigid, they will nevertheless affect the accuracy with which its $\mathbf{J} 2000.0$ equinox and equator can be realized. And the inertial properties of FK5 are not at present known to the accuracy needed for Hipparcos. The IAU is in the process of establishing a non-rotating reference frame through a set of extragalactic objects. A working group is in the process of selecting these objects. The frame can at present be established only by radio interferometry (VLBI) but the emphasis is to be on the selection of sources having suitable optical 
counterparts for making the frame accessible to optical astrometry. It is vital that the Hipparcos Catalogue be linked to this new standard frame. A selection of sources is illustrated by McCarthy et al. (1991, Fig. 1), and many of these sources feature in the link programmes to be described here.

\section{Linking the Hipparcos Catalogue}

Reviews of the proposed methods have been published (Argue $(1989,1991)$ ) but during the time that has elapsed since these papers were written, many important changes have taken place.

\subsection{THE SUPER-HIGH PRIORITY PROGRAMMES}

2.1.1. Hubble Space Telescope. The problems that have arisen with this telescope have created the need for drastic modifications to the programme. The 1989 review referred to 90 extragalactic radio sources linking 166 Hipparcos stars: the latter had been tested for speckle purity as required for the Fine Guidance Sensors by which Hubble carries out its astrometry, and had been entered into the Hipparcos Input Catalogue with super-high priority. Most of these must now be discarded because the limiting brightness for the Fine Guidance Sensors has been changed from $17^{m}$ to a provisional estimate of $15^{\mathrm{m}}$. The new selection will need to contain many objects that are not radio sources of astrometric quality, simply because bright sources are not available in such large numbers, and a further restriction will be that, since Hipparcos is already in orbit (since 1989), the link stars can now be selected only from those already in the Input Catalogue. The object and its associated star must not be separated by more than $18^{\prime}$ to fit into the same 'pickle' of the Sensor. With the new selection there will not be enough VLBI sources to make the optical-toradio alignment, but the rotation of the Hipparcos frame relative to the extragalactic objects can be stopped.

Originally the accuracy specified for Hubble was $0^{\prime \prime} .002$ for the relative objectstar separation and the same for its annual rate of change, so that with 90 pairs the predicted accuracies were $0^{\prime \prime} .001$ for alignment and $0^{\prime \prime} .002 y r^{-1}$ for relative rotation (Froeschlé and Kovalevsky (1982)). At present it is not possible to give the corresponding figures for the amended programme.

2.1.2. Radio stars. Radio stars in principle provide a neat and accurate method of linking. Optically they may be very bright, and 185 have been selected for the Input Catalogue at super-high priority. They are not extragalactic, but their radio positions are measured relative to to the VLBI extragalactic frame. There are serious complications which have been reviewed (Argue 1989). Given radio positions, annual proper motions and parallaxes of accuracy $0^{\prime \prime} .002$ for 30 stars, the accuracy of the link would be $0^{\prime \prime} .0006$ for alignment at epoch, and $0^{\prime \prime} .0009 \mathrm{yr}^{-1}$ for rotation. Only about 33 have been measured by radio astrometry up to the present time (Walter et al. (1990); Lestrade et al. (1991); de Vegt et al. (1991)). However, developments are being made rapidly, and by 1995 it is anticipated that a significant link will be made. 
TABLE I

Link quality predicted for 1995

\begin{tabular}{lll}
\hline Programme & Alignment & Rotation \\
\hline Radio Stars (30) & $0^{\prime \prime} .0006$ & $0^{\prime \prime} .0009 y r^{-1}$ \\
Hubble (50 QSOs:) & $0^{\prime \prime} .0012:$ & $0^{\prime \prime} .0024: y r^{-1}$ \\
Hamburg/USNO/CSIRO & & \\
(400 QSOs) & $0^{\prime \prime} .0010$ & $0^{\prime \prime} .0001 y^{-1}$ \\
CONFOR (200 QSOs) & $0^{\prime \prime} .0014$ & $0^{\prime \prime} .0001 y r^{-1}$ \\
Bonn (10 QSOs) & & $0^{\prime \prime} .0009 y r^{-1}$ \\
Tautenburg & & $0^{\prime \prime} .0005-$ \\
& & $0^{\prime \prime} .0010 y r^{-1}$ \\
Lick/Yale-San Juan & & $0^{\prime \prime} .00007 y r^{-1}$ \\
\hline
\end{tabular}

\subsection{GROUND- BASED OPTICAL PROGRAMMES}

2.2.1. Link to QSOs. An intensive programme of photographic astrometry of QSOs over the whole sky is being carried out jointly at Hamburg, USNO and CSIRO (Johnston et al. (1991)). The aim is to link 400 sources over the entire sky, and is due for completion in 1992. The Russian Programme CONFOR with 200 sources at $\delta>-26^{\circ}$ is also due for completion in 1992 (Kumkova et al. (1991). These programmes will be very powerful in bringing the frames into coincidence at J2000.0 with high accuracy, viz $0^{\prime \prime} .001$ for positions and $0^{\prime \prime} .0001 \mathrm{yr}^{-1}$ for a 20 -year baseline for the proper motions, for 400 sources.

A programme at Bonn entitled 'Fictitious Proper Motions of Quasars', using old plates as well as new, yielded $0^{\prime \prime} .0015 y r^{-1}$ in a preliminary test on four QSOs, with the prospect of an extension to 10 (Brosche et al. (1991)).

2.2.2. Ground-based absolute proper motion programmes. The Lick NPM $(\delta>$ $-23^{\circ}$; Klemola (1990)) and the Yale-San Juan SPM (Van Altena et al. (1990)) proper motion surveys of stars relative to galaxies, have 19,000 and 12,000 stars respectively in common with Hipparcos. The formal error for these surveys combined ought to be as low as $0^{\prime \prime} .00007 y r^{-1}$, a figure reflecting the large number of Hipparcos stars in these surveys, and ignoring zonal errors.

A programme of proper motions of stars with respect to galaxies being carried out with the Tautenburg Schmidt aims at 500 link stars in 25 fields, giving a link accuracy of $0^{\prime \prime} .0005$ to $0^{\prime \prime} .0010 \mathrm{yr}^{-1}$ (Scholz and Hirte (1991)).

\section{Summary and conclusion}

Given realistic expectations for the outcome of the above programmes, the accuracies predicted for the link for 1995 are given in Table 1. These figures indicate that, although the target quality will not be achieved, viz one-tenth of the nominal accuracy for a single star, nevertheless an acceptable quality can be expected. 


\section{Acknowledgements}

The selection of link stars for the Input Catalogue has been the responsibility of a Working Group consisting of:

A.N. Argue (Cambridge; Chairman); P. Brosche (Bonn); Chr. de Vegt (Hamburg); R.L. Duncombe (Austin); P.D. Hemenway (Austin); A.R. Klemola (Lick); J. Kovalevsky (Grasse); J.-F. Lestrade (Paris); C.A. Murray (c/o RGO); G. Ruben (Potsdam); H.G. Walter (Heidelberg); G.L. White (CSIRO ATNF, West Sydney). Although not formally members, the cooperation is gratefully acknowledged of: D.L. Jauncey (CSIRO); I.I. Kumkova (Leningrad); L.V. Morrison (RGO); R.A. Preston (JPL); Y. Réquième (Bordeaux) and R.-D. Scholz (Potsdam).

\section{References}

Argue, A.N.(1989) 'The Link to Extragalactic Objects', in M.A,C. Perryman and C. Turon (eds.), The Hipparcos Mission, ESA SP-1111 Vol. II, pp. 199-211.

Argue, A.N. (1991) 'Hipparcos: its link to an extragalactic reference frame', in J.A. Hughes, C.A. Smith and G.H. Kaplan (eds.), Proc. IAU Coll. 127 Reference Systems, USNO, Washington D.C., pp. 63-67.

Brosche, P., Ducourant, C., Galas, R., Geffert, M. and Karafistan, A (1991) 'Quasar link conditions for Hipparcos', A\&A 245, pp. 669-675.

Froeschlé, M., and Kovalevsky, J. (1982) 'The Connection of a Catalogue of Stars with an Extragalactic Reference Frame', A\&A, 116, pp. 89-94.

Johnston, K.J., Russell, J.L., de Vegt, Chr., Zacharias, N., Hindsley, R., Hughes, J., Jauncey, D.L., Reynolds, J.E., Nicholson, G. and Ma, C. (1991) 'The extragalactic radio/optical reference frame', in J.A. Hughes, C.A. Smith and G.H. Kaplan (eds.), Proc. IAU Coll. 127 Reference Systems, USNO, Washington D.C., pp. 123-9.

Klemola, A.R. (1990) 'Proper Motions with respect to the Extragalactic Reference Frame', in J.H. Lieske and V.K. Abalakin (eds.), Proc. IAU Symp. No. 141, Inertial Coordinate System on the $S k y$, Kluwer pp. 407-417.

Kumkova, I.I., Tel'njuk-Adamchuk, V.V., Irkaev, B.N., Inasaridse, R. Ya., Bashtova, L.I. and Dick, W.R., (1991) 'The linkage between radio and optical coordinate systems: program CONFOR', in J.A. Hughes, C.A. Smith and G.H. Kaplan (eds), Proc. IAU Coll. 127 Reference Systems, USNO Washington D.C., pp. 120-122.

Lestrade, J.-F., Preston, R.A., Gabuzda, D.C. and Phillips, R.B. (1991) 'VLBI aastrometry of the Hipparcos link radio stars', in J.A. Hughes, C.A. Smith and G.H. Kaplan (eds.), Proc. IAU Coll. 127 Reference Systems, USNO Washington D.C., p. 292.

McCarthy, D.D., Russell, J., Archinal, B., Carter, M.S., Florkowski, D., Holdenried, E., Johnston, K. and Yao, Z.-G. (1991) 'The extragalactic reference frame', in J.A. Hughes, C.A. Smith and G.H. Kaplan (eds.), Proc. IAU Coll. 127 Reference Systems, USNO Washington D.C., pp. 309-313.

Morrison, L.V., Argyle, R.W., Réquième, Y., Helmer, L., Fabricius, C., Einicke, O.H., Buontempo, M.E., Muiños, J.L. and Rapaport, M (1991) 'Evidence of systematic errors in FK5', in J.A. Hughes, C.A. Smith and G.H. Kaplan (eds.), Proc. IAU Coll. 127 Reference Systems, USNO, Washington D.C., p. 318.

Scholz, R.-D. and Hirte, S. (1991) 'Tautenburg proper motion fields', Astron Nachr. 312, pp 45-48.

Van Altena, W.F., Girard, T., López, C.E., López, J.A. and Molina, E., (1990) 'The Yale-San Juan Southern Proper Motion Program', in J.H. Lieske and V.K. Abalakin, Proc. IAU Symp. No. 141 Inertial Coordinate System on the Sky, pp. 419-426.

de Vegt, Chr., Zacharias, N., Johnston, J.K. and Hindsley, R. (1991) 'Improving the reference frame by radio and optical astrometry of radio stars', in J.A. Hughes, C.A. Smith and G.H. Kaplan (eds), Proc. IAU Coll. 127 Reference Systems, USNO, Washington D.C., pp. 246-9.

Walter, H.G., Hering, R. and de Vegt, Chr. (1990) 'An astrometric catalogue of radio stars', A\&AS, 86, pp. 357-393. 